\title{
Зависимость продольно-поперечного расщепления экситона в квантовой яме от внешнего однородного электрического поля
}

\section{Логинов Д.К.}

Санкт-Петербургский государственный университет, 198504, Санкт-Петербург, Старый Петергоф, ул. Ульяновская, 3

DOI 10.34077/Semicond2019-169

Настоящая работа посвящена точному численному решению задачи об экситоне в сильном однородном электрическом поле в квантовой яме (КЯ). Трудность анализа экситонных состояний во внешнем электрическом поле состоит в том, что точное аналитическое решение уравнения Шредингера для такой задачи в общем виде не известно. В слабом поле для расчета экситонных состояний используют теорию возмущений $[1,2]$. Если поле нельзя считать слабым, то применение этого подхода становится некорректным. Для больших полей задача на собственные значения и собственные функции экситонного гамильтониана решается в рамках квазиклассического приближения [1,2]. Однако, это приближение корректно описывает только состояния с большим главным квантовым числом $n>>$ [2]. Этот подход некорректен для описания

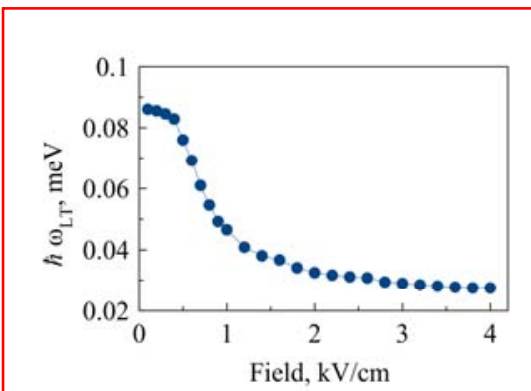

Pис.1. - Зависимость продольно-поперечного расщепления экситона от внешнего однородного электрического поля в КЯ на основе GaAs/AlGaAs шириной $120 \mathrm{~nm}$. основного экситонного состояния $(n=1)$, которое зачастую вносит наибольший вклад в оптические спектры, наблюдаемые в эксперименте.

В нашей работе была решена задача на собственные значения и собственные функции для основного состояния экситона в электрическом однородном поле в КЯ на основе $\mathrm{GaAs} / \mathrm{AlGaAs}$ гетероструктуры, выращенной вдоль направления [001]. Ширина КЯ полагается равной $120 \mathrm{~nm}$. Электрическое поле, $F$, было направлено нормально к плоскости КЯ, строго вдоль [001]. Мы переписали гамильтониан в параболических координатах и разделили его на два одномерных дифференциальных уравнения, в соответствие со стандартной процедурой $[1,2]$. Полученные уравнения были представлены в виде разностных схем и решались трехдиагональным методом [4]. Были определены собственные волновые функции экситона и его энергия связи при различных $F$. Это позволило вычислить зависимость от поля продольно-поперечного расщепления экситона $\hbar \omega_{\mathrm{LT}}$ , описывающего интенсивность взаимодействие экситона со светом [3]. Результаты вычислений представлены на рисунке. Как можно видеть, $\hbar \omega_{\mathrm{LT}}$ быстро спадает с ростом поля и выходит на почти постоянное значение при $F>3 \mathrm{kV} / \mathrm{cm}$. Таким образом, спектральные особенности, обусловленные основным состоянием экситона $(n=1)$, в КЯ, будут терять интенсивность с ростом поля.

[1] D. F. Blossey, Rev. B 2, 3976 (1970).

[2] М. Д. Ландау, М. Е. Лифшии, Теоретическая физика. т. ІІІ. Квантовая механика. Нерелятивистская теория. Наука. Москва. (1989).

[3] E. L. Ivchenko, Optical Sp ectroscopy of Semiconductor Nanotstructures, Alpha

Science, Harrow, (2005).

[4] A.A. Samarskii, The Theory of Difference Schemes, Pure and Applied Mathematics.

A Series of Monographs and Textb o oks, CRC Press, (2001). 